\title{
Strates
}

STRATES Matériaux pour la recherche en sciences sociales

14 | 2008

Espaces du quotidien

\section{Sur la construction des territoires urbains quotidiens en Algérie}

Bouziane Semmoud

\section{(2) OpenEdition}

1 Journals

Édition électronique

URL : http://journals.openedition.org/strates/6665

DOI : $10.4000 /$ strates.6665

ISSN : $1777-5442$

Éditeur

Laboratoire Ladyss

Édition imprimée

Date de publication : 1 janvier 2008

Pagination : 39-54

ISBN : 0768-8067

ISSN : 0768-8067

Référence électronique

Bouziane Semmoud, "Sur la construction des territoires urbains quotidiens en Algérie ", Strates [En ligne], 14 | 2008, mis en ligne le 04 mars 2013, consulté le 08 septembre 2020. URL : http://

journals.openedition.org/strates/6665; DOI : https://doi.org/10.4000/strates.6665

Ce document a été généré automatiquement le 8 septembre 2020

Tous droits réservés 


\title{
Sur la construction des territoires urbains quotidiens en Algérie
}

\author{
Bouziane Semmoud
}

\section{NOTE DE L'ÉDITEUR}

Les cartes et photos sont de l'auteur.

\section{NOTE DE L'AUTEUR}

Avec la collaboration de Ali Aït-Amirat, géographe aménageur, Oran.

\section{Introduction}

1 En dépit de la multiplication des travaux sur les villes d'Algérie, quelques idées reçues jalonnent encore partiellement la connaissance qu'on peut avoir de la vie urbaine de ce pays en particulier des pratiques et des représentations de leurs habitants. Il en est ainsi de leur supposée inadaptation irréductible à l'habitat collectif qui engendrerait des comportements irrationnels voire déviants, comme du malaise que ressentirait l'Algérien des grands ensembles à la fois dans son logement et dans son environnement, cause de la faible territorialisation qui y est observée. L'hiatus entre le mode d'habiter algérien profondément marqué du sceau du Culturel, et le mode de planification du logement et plus généralement du cadre urbain seraient les facteurs principaux du déséquilibre et du malaise.

2 L'objectif initial du présent article est de voir en quoi les valeurs culturelles imprégnent voire déterminent les pratiques spatiales et les représentations. L'examen des pratiques de toutes sortes (économiques, spatiales...) semble traduire d'emblée une imbrication enchevêtrée de la tradition et de la modernité, laquelle tient à un état de transition qui 
puise sa source dans les évolutions contradictoires de la société et des influences extérieures. Dans quelle mesure les pratiques spatiales articulent-elles stratégies matérielles et arsenal culturel mobilisé par les habitants ou les groupes sociaux? Cette interrogation est au cœur de l'investigation sur les pratiques d'habiter et de voisiner dans les nouvelles périphéries urbaines, et les types de rapports tissés entre les habitants et les espaces publics.

Le soin porté par les Algériens à leur logement révèle la prééminence du dedans sur le dehors et tient autant à un repli très fort sur la famille dans un contexte urbain souvent considéré comme hostile, qu'à la médiocrité de l'espace public en tant que lieu de sociabilité (Semmoud, 2004). Différents modes de territorialisation n'en sont pas moins visibles, tantôt solides tantôt déconstruits dans les anciens tissus ou en gestation plus ou moins avancée dans les nouvelles périphéries. C'est dans ces dernières, grands ensembles d'habitat collectif ou lotissements - notamment populaires - que l'on a tenté de cerner le processus de territorialisation en marche, avec des populations venues de divers horizons sociaux et géographiques, d'apprécier la place qu'y tient l'expérience de la vie urbaine commune face aux différents liens de type professionnel ou communautaire, bref de voir comment se construit un territoire urbain au quotidien.

Les enquêtes menées lors de l'été 2006 à Oran, ont été conduites sur le mode des entrevues directes parfois complétées par des cartes mentales. Les entretiens ont cherché à exhumer, dans les discours, les éléments qui dominent dans l'organisation du territoire du quotidien, interroger certaines catégories sémiologiques : la houma, le haï ou quartier, la cité (au sens de grand ensemble), la ville... L'agglomération oranaise (figure 1), qui comptait 968000 habitants au recensement de 1998, en rassemble sans doute plus de 1100000 en 2006. Comme les autres grandes villes d'Algérie, elle connaît une extension importante de son périmètre sur lequel se redistribue une population dont la croissance encore soutenue ne dépasse toutefois guère les $2 \%$ par an. Le choix des terrains et enquêtes a été porté sur deux secteurs urbains d'une certaine importance, qui renvoient des images contrastées, mais où la population est partiellement libérée du souci de se loger ${ }^{1}$. La question est de savoir si, une fois la contrainte du logement levée, la population produit de la sociabilité, se territorialise, ou reste dans une logique de mobilité constante, de tracé d'itinéraires. 
Figure 1. Oran et ses extensions. Carte de situation des secteurs de Maraval et de Chteibo.

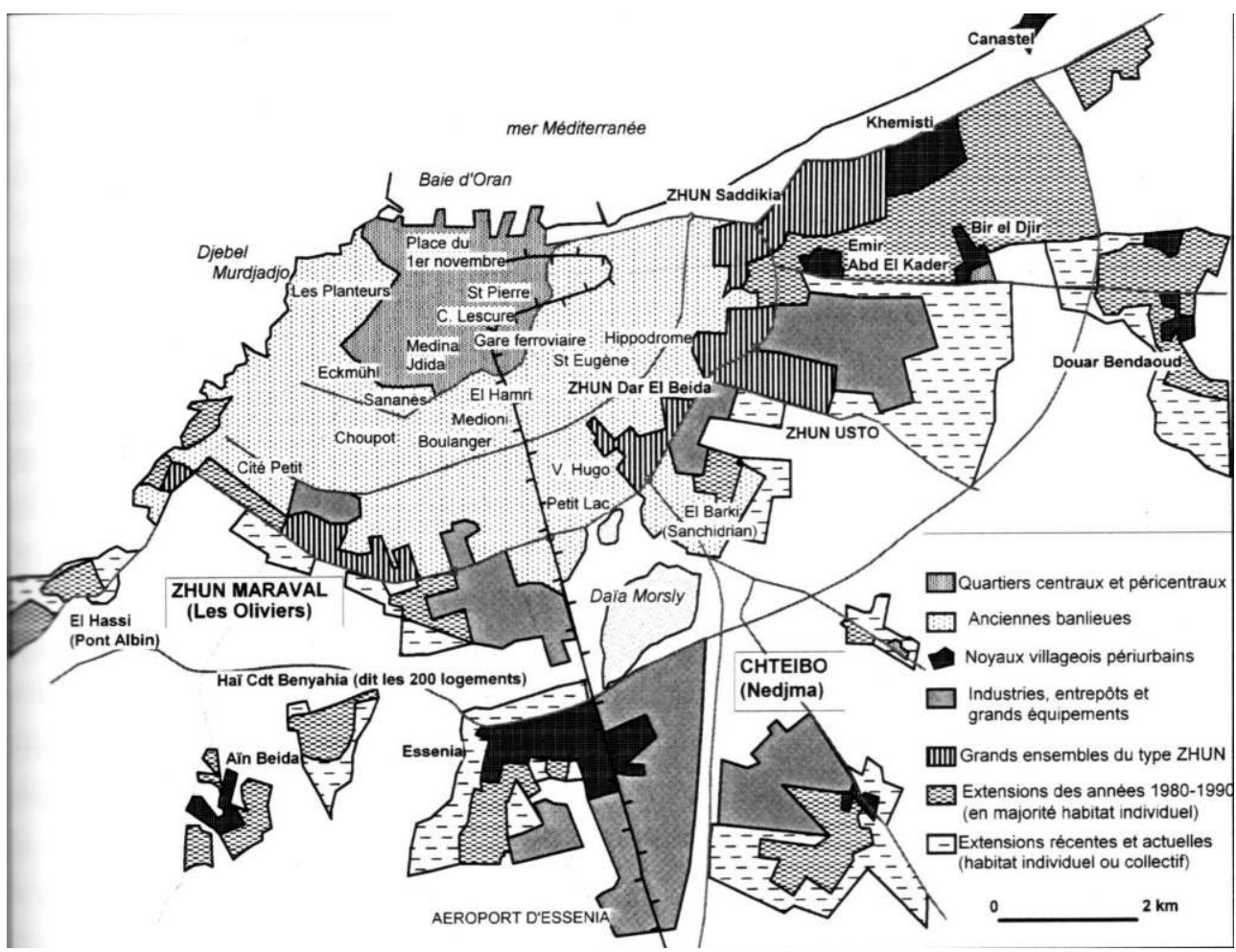

Les enquêtes ont été réalisées dans le secteur à dominante grands ensembles, dit de Maraval (figure 2) et situé à la lisière sud de la ville proprement dite. Il regroupe des HLM de la fin de l'ère coloniale (Les Glycines, la Cité Radieuse) et l'immense ZHUN (zone d'habitat urbain nouveau) qui en porte le nom. L'image du secteur est globalement positive produisant une certaine attractivité. On s'est intéressé en particulier à deux unités de voisinage dont le contenu est homogène (enseignants du primaire) ou hétérogène (association de personnel de l'enseignement supérieur, de relogés, etc.). 
Figure 2. Le secteur dit de Maraval.

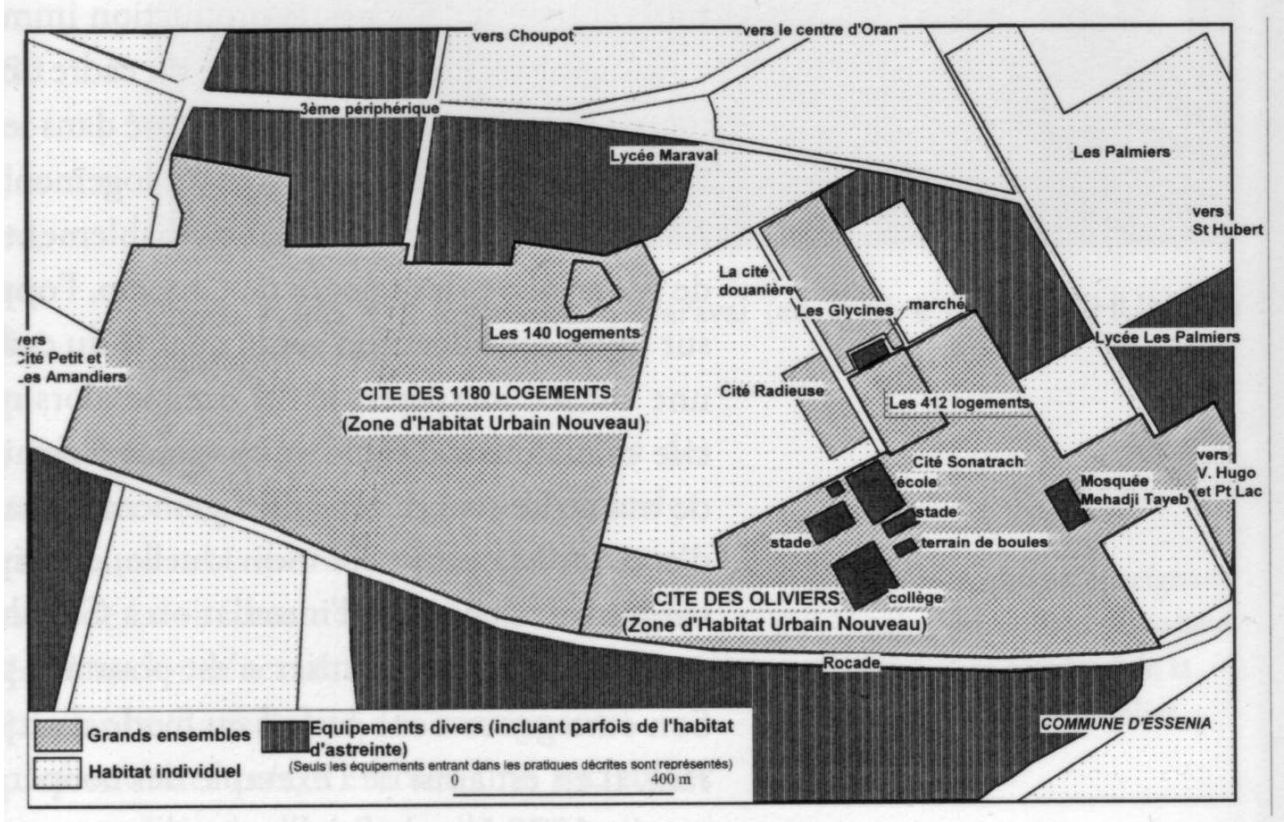

6 Les enquêtes ont été également menées dans l'agglomération périurbaine de Nedjma, connue communément sous le nom de Chteibo. Située dans la commune périphérique de Sidi Chahmi, au sud-est de la ville, elle est passée, en vingt ans du statut de zone industrielle classée zone éparse, à peine peuplée de 220 personnes en 1977, puis 4300 en 1987 à une véritable agglomération de 50000 habitants, constituée de lotissements essentiellement populaires tracés au cordeau. C'est un immense «quartier-ville » de recasement né de la nécessité de reloger des familles déguerpies d'Oran puis, à partir de 1989, des grandes opérations de résorption de l'habitat précaire. Son image négative ne lui interdit pas d'être attractive, du fait d'une très forte intégration économique à l'agglomération et d'une grande offre foncière initialement accessible par des démarches simplifiées au maximum. Les entrevues y ont été facilitées par la collaboration de Aït Amirat, géographe aménageur qui y avait déjà effectué les enquêtes de régularisation pour le compte de la municipalité de Sidi Chahmi ${ }^{2}$.

7 Une vingtaine d'entretiens structurés ont été conduits dans chacun des secteurs mais des entrevues avec des gardiens de parking (d'excellents observateurs) ou des commerçants, et plusieurs discussions collectives ont permis de croiser les discours voire d'en souligner les contradictions. Enfin, des sorties sur le terrain avec des collègues en ont éclairé le contenu et les processus ${ }^{3}$.

\section{Les territoires du quotidien : du voisinage au quartier}

L'examen des modes d'accès au sol ou au logement révèle que jusqu'à la multiplication récente des filières de production immobilière, il n'y avait pas, pour la majorité des Algériens, de choix possible du cadre de vie, de l'environnement. Le citoyen était tenu d'accepter le logement proposé dans le cadre de l'attribution par l'entreprise qui l'employait ou par les services du logement social sous peine de manquer une occasion qui ne se représenterait probablement pas. Qu'il s'agisse des directeurs d'école de Maraval ou des relogés de Chteibo, l'opportunité d'accès au logement l'emportait sur 
toute considération sur la qualité du cadre de vie. «L'attribution du logement fut une chance inespérée, je l'ai occupé alors que la cité était encore en chantier ", précise ce directeur d'école qui a vécu à Eckmühl et y a vu naître ses trois garçons, dans de mauvaises conditions de logement, cohabitant avec la famille élargie. "La chance d'accéder à la propriété individuelle, en dépit de la médiocrité du cadre de vie », est l'argument majeur de l'installation à Chteibo, avancé par un petit transporteur.

La houma ou quartier n'est pourtant pas une catégorie complètement absente. Son émergence tient parfois au mode d'acquisition des logements ou des lots de terrain. Il en est ainsi de l'exemple des coopératives immobilières initiées au milieu des années 1970 à seule fin d'inciter les usagers à prendre en charge la réalisation de leur propre logement. La phase d'accession à la propriété du sol, excessivement longue, est parsemée de réunions régulières, de démarches, voire de lobbying auprès des pouvoirs publics... Elle élargit les liens de sociabilité aux membres de la famille des coopérateurs, liens préexistants entre ces derniers du fait de la constitution des coopératives par corps professionnels (avocats, enseignants, cadres administratifs, douaniers, gendarmes, commerçants...).

10 Il en a été de même, jusqu'à très récemment, des grands ensembles dont les logements étaient pour l'essentiel répartis selon des quotas aux différents secteurs économiques : des blocs voir des cités entières sont habités par des enseignants, des gendarmes ou des salariés de telle entreprise nationale industrielle ou de services, impliquant souvent une relative homogénéité culturelle ou/et sociale. Il n'en est plus de même aujourd'hui, sauf marginalement, avec les différentes formes de promotion immobilière et d'accès à la propriété. Cette situation a pu souvent souder les habitants autour de la gestion de l'espace public laissé à l'abandon, ou de sa protection contre les déprédations extérieures : dépôt d'ordures sauvage, envahissement des caves ou des buanderies... ou face aux multiples convoitises sur les espaces libres.

11 Le secteur de Maraval est composé entre autres par de petites «cités » initialement dévolues à des corps professionnels : la Cité Sonatrach et la Cité Radieuse pour les enseignants et les agents de l'enseignement supérieur, les 412 logements réalisés par la municipalité d'Oran et dédiés à l'enseignement primaire (photo 1), à côté d'ensembles socialement et professionnellement mixtes (Cité des Oliviers, Cité des 1180 logements). La mobilité les a touchés diversement à la suite de la cession, dès les années 1980, du parc immobilier d'abord, de la libéralisation des transactions immobilières ensuite : si le contenu de la Cité Radieuse et de la Cité Sonatrach a été profondément remanié, celui de la Cité des enseignants du primaire et de la Cité des Oliviers ne l'a été que marginalement. Ainsi deux des plus anciens blocs de cette dernière affichent une grande stabilité et de ce fait une certaine mixité. Sur les huit occupants initiaux d'une des entrées ${ }^{4}$, deux ont cédé très récemment leur logement à des émigrés à l'étranger : le bijoutier qui a accédé à un lot de terrain et les héritiers du postier décédé. Le renouvellement est du même ordre dans la cité des enseignants. 
Photo 1. Les 412 logements à Maraval (en arrière plan, la ZHUN des Oliviers).

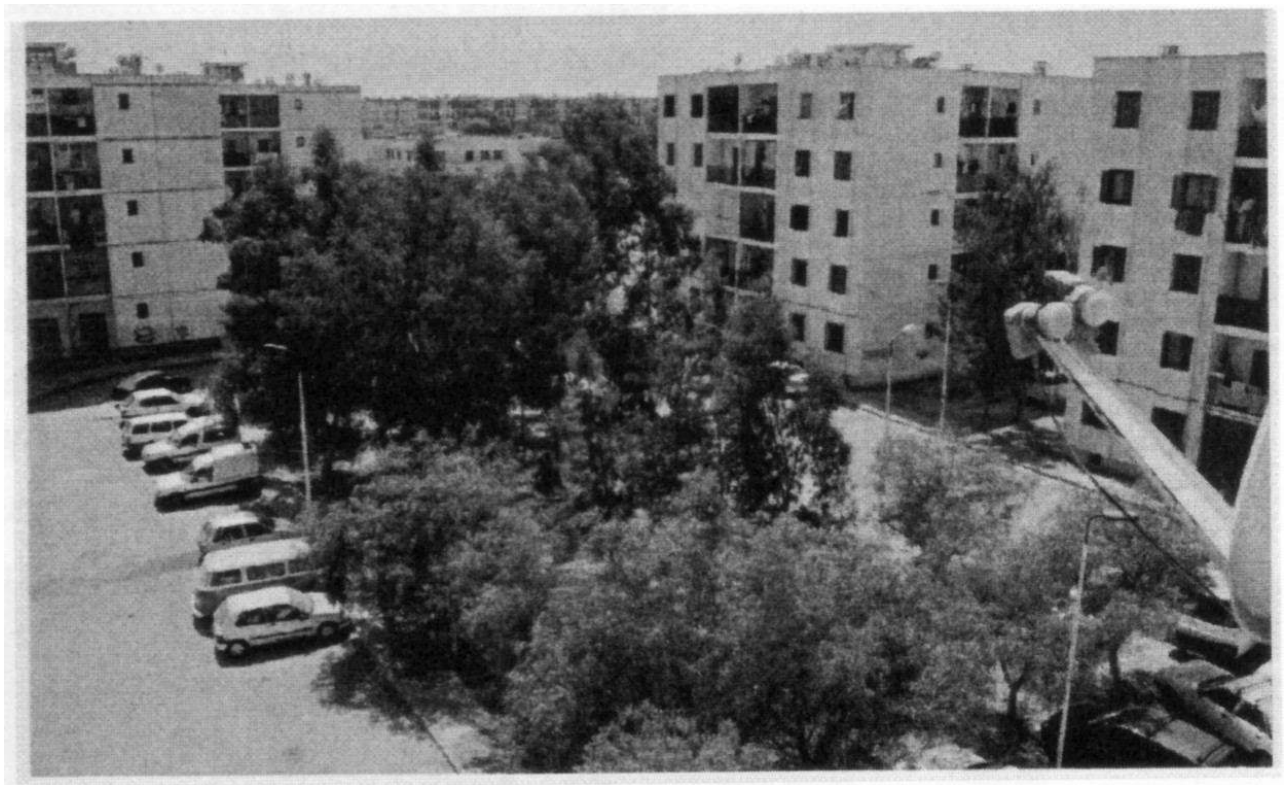

12 L'appartenance à un même corps professionnel crée d'emblée une sociabilité qui, d'habitude mettrait longtemps à émerger. "J'ai retrouvé des collègues avec qui j'avais souvent ou un moment travaillé. Nous avions le même âge, les mêmes habitudes, la même profession, les mêmes origines régionales, nous constituions une communauté (cité en français)", précise un directeur à la retraite. Un grand nombre de ces enseignants sont en effet des directeurs d'école, dont les parents avaient émigré de la région de Tlemcen à Oujda avant ou pendant la guerre d'indépendance (ou y étaient nés), et qui avaient pour certains d'entre eux fréquenté les mêmes établissements scolaires. C'étaient majoritairement des hommes dont les épouses étaient femmes au foyer, ayant donc des ressources équivalentes. Deux collègues, dont un retraité, relatent les conditions dans lesquelles s'est faite l'occupation des trois premiers blocs et qui ont déterminé des liens de solidarité aujourd'hui encore très forts : « Les travaux de finition des logements attribués dans l'urgence ont été réalisés en touiza ${ }^{5}$, et durant les premiers Ramadhan(s), un logement attribué et encore inoccupé, servait à faire des gâteaux par un connaisseur, cédés aux habitants à prix coûtant. Des sorties collectives à la mer ou à la forêt étaient possibles grâce à ceux qui possédaient des voitures... » Le voisinage construit sur l'appartenance professionnelle, s'il est pesant pour certains enseignants parce qu'il prolonge sur le lieu de vie d'éventuels conflits professionnels, est généralement apprécié, parce que porteur de fortes solidarités : «Au décès de mon épouse, l'aide et le soutien de mes collègues et amis voisins furent plus importants que l'appui de ma propre famille", précise le même directeur. Cette cohésion peut se fissurer, si un des éléments vient à manquer : les époux des enseignantes sont moins intégrés, et des sous-groupes peuvent se constituer par origines géographiques (les originaires de Tlemcen, ceux de Relizane...). À la Cité des Oliviers proche, c'est plutôt l'effet des retrouvailles d'amis d'enfance disséminés dans les différents blocs, qui favorise une interconnaissance de moindre intensité.

13 L'exemple de la Cité des 140 logements, réalisée tardivement sur un espace intersticiel de l'ensemble des 1180 logements est significatif du poids des appartenances dans la faible cohésion de l'espace qui accompagne une forte mixité sociale et qui traduit là aussi les modalités d'attribution des logements. C'est un ensemble de quatre blocs de 
cinq étages au contenu différencié dont chacun a une population socialement homogène (à l'exception d'un seul) qui privilégie l'entre soi. Dans le premier, les relogés de l'habitat dégradé du vieux centre de Sidi Lahouari, du quartier périphérique d'El Barki, vivent repliés sur eux-mêmes avec de fortes inter-relations familiales. Ils se sont nettement démarqués des autres habitants en s'appropriant le devant de l'immeuble ainsi qu'une portion de l'espace public central, interdisant de fait le passage aux autres usagers et en créant une ruelle ombragée entièrement privative (photo 2). Le second bloc est occupé par les relogés de Saint Eugène, une ancienne banlieue, qui y ont reconstitué les liens de quartier antérieurs, au point que leurs enfants ont formé une équipe de football commune avec ceux qui sont restés sur place. Des universitaires occupent le troisième immeuble, tissant un réseau avec d'autres quartiers d'Oran ou d'autres villes dont ils sont originaires. L'espace de voisinage est ainsi éclaté au profit de relations en réseau avec des lieux extérieurs, la seule marque de solidarité étant la résidentialisation menée contre l'agression extérieure : deux des trois voies d'accès ont été obstruées par des piliers ou de grosses pierres, empêchant le passage des automobilistes non riverains alors que la mise en place d'un comité de quartier demeure une velléité.

Photo 2. Appropriation-aménagement en ruelle bordée d'espaces verts aux 140 logements de la Cité des 1180 logements.

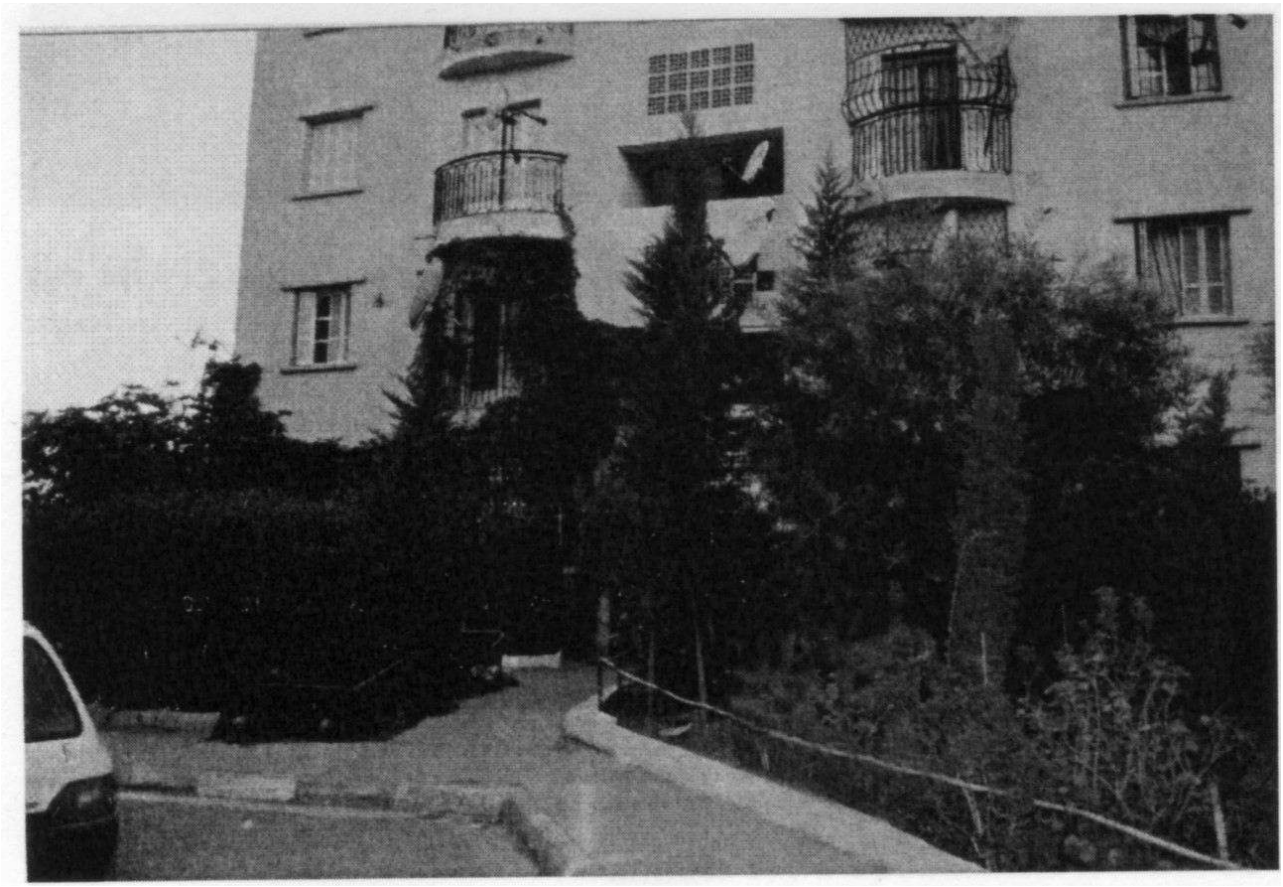

14 À l'inverse des grands ensembles dont le peuplement a été exclusivement le produit de la mobilité résidentielle, celui de Chteibo résulte de l'interférence entre des-serrement urbain et exode rural de nature essentiellement sécuritaire. Les statistiques disponibles ne peuvent en fournir une estimation satisfaisante $d u$ fait des nombreuses et incessantes transactions (entre 20 et $50 \%$ selon les lotissements) lesquelles ont fini par « informaliser» un grand nombre de lotissements initialement légaux. Si le mode de cession des lots a favorisé quelquefois des regroupements par corps professionnels (lotissements des éboueurs et des travailleurs de la jumenterie), l'effet d'affinage par les transactions a joué en faveur de rassemblements par origines géographiques, 
notamment pour les néo-urbains, par village, voire par douar. Dans les lotissements enquêtés, certaines rues sont quasiment dominées par des originaires de Ouled 'Aïch ou de Mendès du massif de l'Ouarsenis (wilaya de Relizane) ou d'El Bordj des Monts de Beni Chougrane dans la wilaya de Mascara. Le recensement de 1998 en rend compte pour la dernière décennie: ces communes mais aussi celles de Zemmoura et de 'Ain Tarik également montagneuses, ont été, après le chef-lieu de la wilaya de Relizane, les plus forts pourvoyeurs en migrants, de la commune de Sidi Chahmi, installés en majorité à Chteibo (Prenant, Semmoud, 2006).

Interroger les populations de Chteibo sur leur quotidien paraît incongru tant l'environnement urbain est de mauvaise qualité. La quasi-totalité des bâtisses sont inachevées ou à l'état de chantier, les rues non bitumées sont poussiéreuses ou boueuses à l'exception de l'avenue principale des Mawwaline ${ }^{6}$, l'alimentation en eau n'est pas assurée, le réseau venant d'être refait, et l'assainissement est inexistant; le ramassage des ordures ménagères est très irrégulier, le nettoyage des rues non pris en charge et le bruit des avions atterrissant à ou décollant du tout proche aéroport d'Essenia est assourdissant (photos 3 et 4). En revanche les transports collectifs sont assurés par le secteur privé, quoique dans des conditions de confort médiocres et desservant prioritairement, rentabilité oblige, la ville d'Oran.

Photo 3. La place centrale d'un lotissement à Chteibo en cours d'aménagement en espace vert.

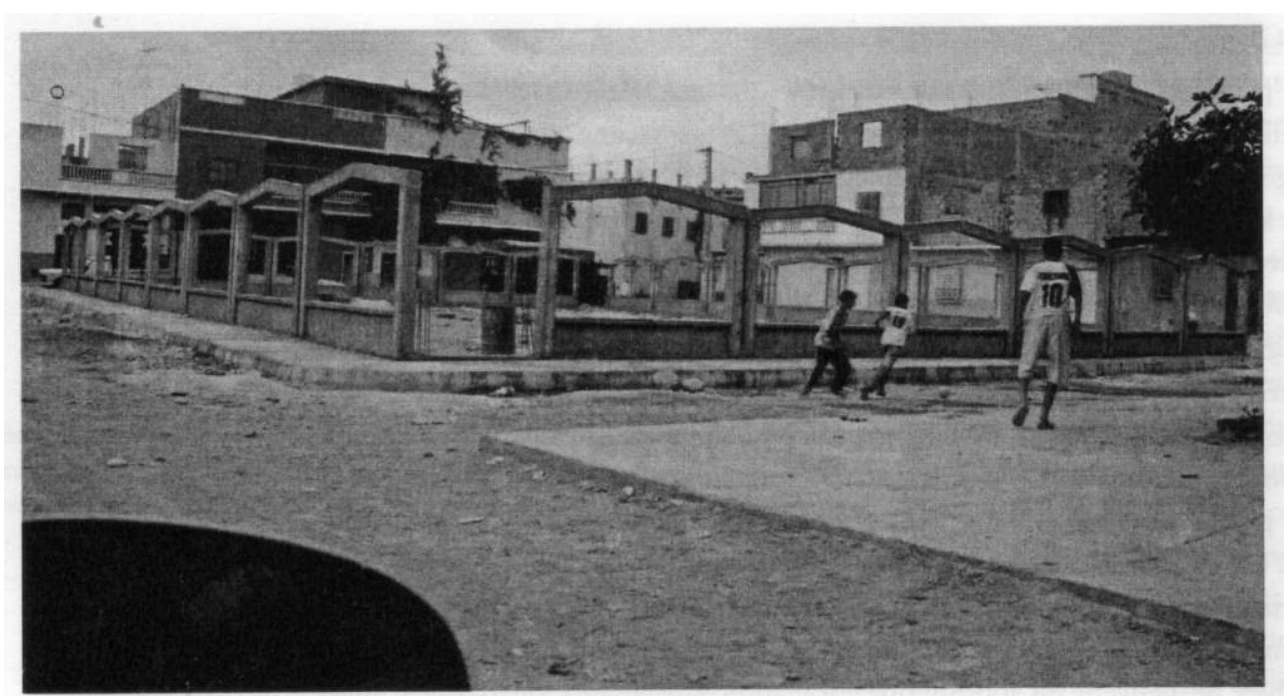


Photo 4. Une rue à Chteibo.

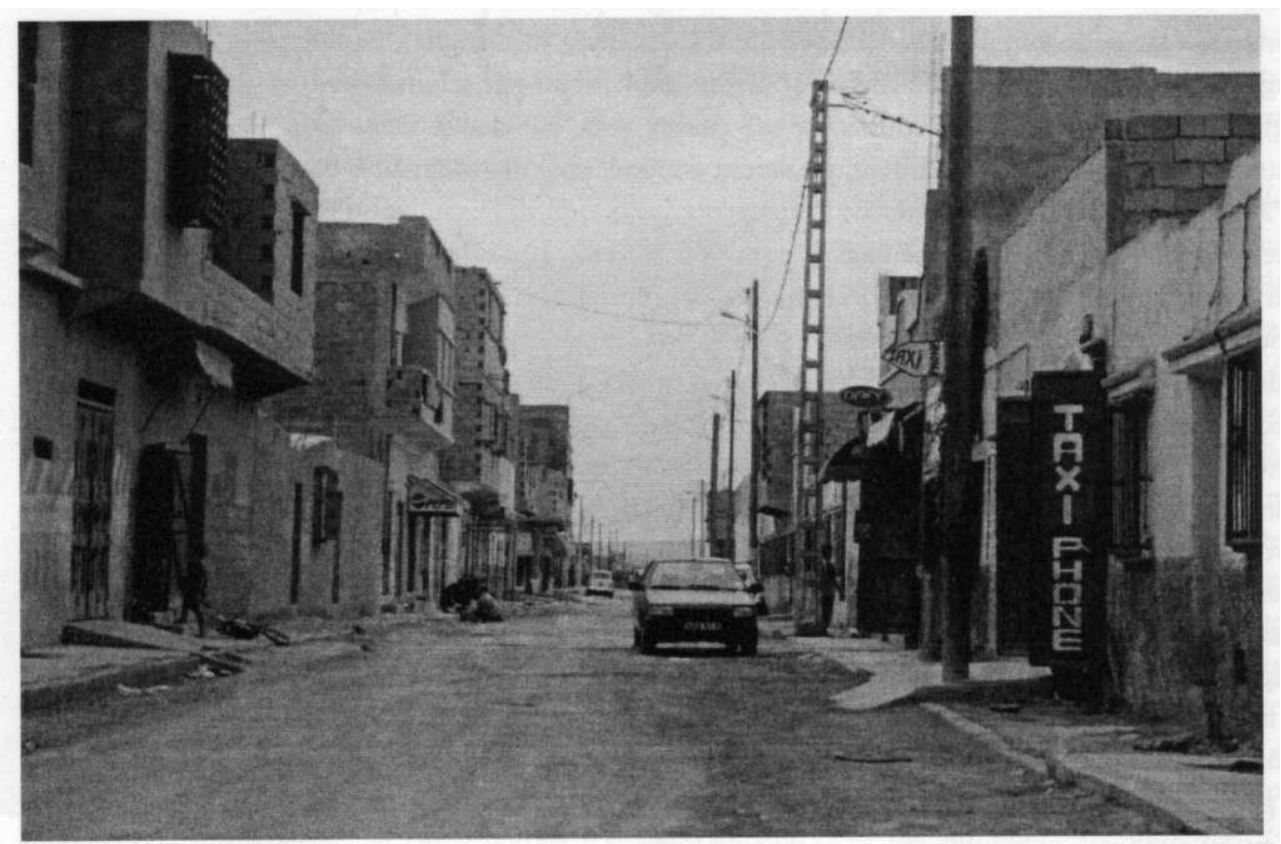

16 À la question principale sur les facteurs d'attractivité, les réponses sont unanimes. En premier lieu c'est le coût des lots ou des maisons, les plus bas de toute la périphérie oranaise ; aujourd'hui encore, malgré la spéculation, l'éventail des prix est tel qu'il permet l'accès des plus défavorisés au sol. En second lieu ce sont les possibilités d'emploi dans le secteur surtout informel mais aussi formel malgré la fermeture récente de plusieurs entreprises publiques et privées. Pour ce transporteur de 40 ans, arrivé d'Ouled Aïch il y a 22 ans à Oran où il a travaillé dans des chantiers en fondant, au village, une famille qu'il ne fait venir qu'en 1995, au plus fort de l'insécurité : «Chteibo m'a offert l'occasion unique de posséder une maison individuelle et la possibilité d'exercer une activité (informelle). Aidé d'un de mes fils, je fais, avec ma camionnette, du transport de matériaux de construction, et d'autres marchandises vendues à Chteibo ${ }^{7}$. Mes deux autres enfants travaillent l'un comme manœuvre dans le bâtiment, l'autre comme vendeur de légumes au marché. Sur la carte mentale qu'il a esquissée, l'espace de travail est dominant: linéaire, il ne comporte ni la direction d'Oran ni celle de Mers el Kébir qu'il évite car susceptibles d'entraîner un contrôle policier.

17 L'ensemble des entretiens révèle que le noyau de vie est structuré autour du couple famille/logement et du lieu de travail. Si pour les urbains desserrés, Chteibo n'est souvent qu'un simple espace-dortoir, pour les néo-urbains, le quotidien se situe essentiellement à Chteibo car le travail s'y trouve partiellement. Chez ces derniers, l'espace vécu est d'abord structuré par l'appartenance au village, ou au douar (dans certains lotissements, près de la moitié des maisons sont occupées par des familles originaires d'un même douar); cette appartenance est revendiquée, recherchée, justifiée par le discours sur l'insécurité (agression, méfiance vis-à-vis des inconnus...), elle est garante d'une confiance mutuelle. «El M'hami yaghlab esseba'a, plus on est nombreux à appartenir au même groupe, plus on se protège mutuellement, plus on fait pression ", soutient un père de famille immigré qui a quitté 0 . Aïch à cause de l'insécurité. "Je n'habiterai jamais à Oran", avance cet autre habitant. Ce dernier, 
arrivé d'El Bordj dès 1986 pour travailler comme journalier dans les entrepôts de matériaux de construction du centre de Chteibo, a fait venir sa famille en 1993. Il cohabite depuis 1999, après six ans de location, avec un frère et trois sœurs également en famille, sur un lot morcelé en deux. Il souhaite une décohabitation dans la proximité. L'appartenance à la même famille est ainsi une réalité prégnante et quand le regroupement ne se réalise pas dans la même rue, il se fait dans le lotissement ou dans le quartier. Cependant, le voisinage tend à tisser des nouveaux liens qui transcendent apparemment l'appartenance tribale : «Je marie bientôt ma fille au fils d'un des voisins habitant la même rue, pourtant originaire de Tissemsilt ${ }^{9}$ ", informe l'interlocuteur originaire de 0 . 'Aïch. Simultanément, le néo-urbain a pour projet, quand il ne l'a déjà mis en œuvre, de surélever son rez-de-chaussée doté de garage, de plusieurs chambres en vue du mariage des garçons «de préférence avec des jeunes femmes issues du village ", reconstituant ainsi la famille élargie et les beni ' $\mathrm{Am}^{10}$, avatar de la tribu.

En réalité, le voisinage ne se substitue pas à l'appartenance aux beni 'Am qui demeure le siège privilégié des alliances matrimoniales, il vient la renforcer et l'élargir. $A$ contrario, cet employé d'une entreprise nationale d'Oran espère marier ses enfants à Oran dont il est issu. Ainsi urbains desserrés et néo-urbains coexistent sans se mélanger, même quand leurs conditions sociales sont proches. La durée semble toutefois amorcer un décloisonnement entre urbains et ruraux, entre régions d'origine : les cas d'alliances matrimoniales qui l'attestent sont rares et passent encore par l'expérience des époux en milieu urbain. Un serveur de café né à Savignon, ancienne banlieue oranaise, épouse une jeune femme originaire de H'madna, un village de la wilaya de Relizane, dont la famille était passée par le quartier de l'Hippodrome avant de s'installer à Chteibo en 1996. Ce gérant d'une petite boulangerie, issu d'Aïn Touta, petite ville des Aurès, est d'abord manœuvre à Oran puis à Chteibo au début des années 1990, avant d'épouser une jeune femme d'un "village» périurbain et de s'installer sur un lot.

\section{Les habitants entre territoire et réseau}

Le faible enracinement dans les grands ensembles est certes réel mais il est à nuancer ${ }^{11}$. Il s'y développe une articulation originale mais diverse avec les anciens quartiers de résidence. Plusieurs habitants de la cité des 412 logements se sont installés à partir de 1993, après avoir presque toujours effectué plusieurs mobilités depuis le centre ville ou depuis d'anciennes banlieues, là où, enfants ou adolescents, ils s'étaient installés avec leurs familles à leur retour du Maroc dans les années 1960. Tel directeur d'école à Cité Petit, une ancienne banlieue, a habité à Saint Pierre, puis à la Cité Lescure, avant de s'installer en 1982 dans les tous premiers blocs de la ZHUN des Oliviers puis, en 1996, à la cité des 412 logements. Tel autre, a grandi à la place du 1er novembre avant de s'installer, à son mariage, à la Cité Lescure, puis en 1996 dans l'actuelle Cité. Un quatrième a habité successivement à Dar el Beïda, à l'Hippodrome et à Victor Hugo où, après s'être marié, il a cohabité avec ses parents. Deux autres enfin ont résidé trente ans, l'un à Saint Eugène, l'autre à Eckmühl, où ils ont vu naître leurs enfants au sein de la famille élargie. Dans tous les cas, le logement familial est conservé, occupé par des parents âgés ou repris par un frère ou une sœur. Hormis des cas particuliers de rupture définitive avec le quartier d'origine, des liens très étroits sont entretenus sous la forme de visites familiales régulières, une à plusieurs fois par semaine, voire quotidiennes en 
cas de proximité du lieu de travail, de relations suivies d'ancien voisinage et d'amitié, parfois d'habitudes d'achat ou de recours à des services. C'est souvent une véritable multiterritorialisation qu'ils vivent et assument, au point que certains interviewés se sentent appartenir à deux quartiers à la fois. L'ancrage dans l'ancien quartier peut être exclusif dans le cas d'une installation récente: ainsi pour ce couple de directeurs d'écoles qui, à son arrivée de Nédroma, petite ville de la wilaya de Tlemcen, a habité pendant trente-cinq ans au centre ville avant d'emménager en 2005, les 412 logements ne constituent qu'une cité dortoir ; ils dirigent des écoles dans leur ancien quartier, y maintiennent la scolarité de leurs enfants et y font leur marché. Il est aussi exclusif dans le cas d'une faible mobilité antérieure et la nostalgie d'une vie de quartier « citadine » où : «J'étais connu comme le fils de Hadj..., aujourd'hui, nous vivons dans l'anonymat le plus total », regrette ce directeur d'école.

La vie de quartier n'est ainsi pas seulement interconnaissance mais aussi reconnaissance. Il est, de ce point de vue, une différence notable entre les secteurs urbains de la ville-centre et les extensions récentes notamment les grands ensembles et les lotissements, mais aussi l'habitat informel populaire. Dans les premiers, l'équipement hiérarchisé contribue à structurer les espaces en coquilles emboîtées dans lesquelles se reconnaissent les habitants y compris ceux qui ont depuis emménagé dans les nouvelles banlieues ${ }^{12}$. La durée, même courte, est productrice d'entités territoriales vécues. Des quartiers comme Sidi Lahouari, Mdina Jdida, Sananès, Eckmühl, El Hamri, Médioni, Choupot, Cité Petit... ont acquis une forte identité. Les extensions récentes éprouvent, à l'inverse, du mal à s'ériger en territoires du fait même de leur retard en équipements structurants. Le cas extrême d'attachement est celui de l'habitant de Choupot qui sous-loue le logement acquis dans une ZHUN de l'Est de la ville pour prendre une location dans son propre quartier.

Il semble malgré tout qu'une certaine vie de quartier se met en place dans les nouvelles périphéries, comme dans la ZHUN de Maraval à laquelle appartient l'ensemble des 412 logements, notamment autour d'équipements réalisés au coup par coup: deux mosquées, deux terrains de football, un terrain de pétanque, et un café au rayonnement important, outre un tissu dense de petits commerces et de prestataires de services. Elle se met pour partie en place dans l'action. Rappelons que les ZHUN n'étaient pas des procédures d'aménagement dans la mesure où les actions d'équipement relevaient d'acteurs différents du maître d'ouvrage et n'ont suivi que ponctuellement, dans l'urgence, notamment des écoles, des collèges, des annexes d'APC ${ }^{13} \ldots$ Le portefeuille foncier de la ville ayant fondu, les assiettes réservées aux équipements « fantômes » ont été convoitées par des acteurs privés. Dans le cas présent, les habitants ont réagi par la constitution d'associations et de comités de quartier qui ont imposé la réalisation des terrains de football et de pétanque au coup par coup, résistant ainsi aux coups de force répétés d'acteurs privés extérieurs à la ZHUN parfois soutenus par des représentants des pouvoirs publics. Le plus grand des stades est géré par l'association « la Radieuse » qui y organise, outre l'activité sportive locale, des tournois de football à l'échelle de l'ensemble de la ville, avec la participation d'anciennes gloires nationales, animant ainsi le quartier en lui assurant une ouverture nécessaire. De même le terrain de pétanque ou le café sont des lieux de convivialité masculine locale, non moins ouverts sur l'extérieur du fait des tournois spontanés sur le premier et de fréquentation du second par des anciens de la ZHUN habitant de nouveaux quartiers pavillonnaires proches « sans vie », et restés fidèles au quartier, au café comme au hammam. 
22 La gestion des espaces "communs" (ils ne relèvent pas du Public si celui-ci s'en désintéress ${ }^{14}$ ) suscite également des actions communes créatrices de solidarités éphémères telle la plantation de carrés (place centrale des 412 logements, espace vert de La Radieuse...) contre des velléités d'appropriation extérieure, ou durables telle l'organisation d'un parking collectif couvert, et à l'échelle de plusieurs blocs, la gestion des ordures ménagères et des parties communes où des femmes des 412 logements ont pu s'illustrer par des démarches visant à faire nettoyer les caves ou à louer les services de femmes de ménage.

23 La mobilité résidentielle des habitants a diversement agi sur l'espace de fréquentation quotidien des femmes. À Maraval, elle a provoqué un relatif élargissement; elle a en tout état de cause libéré la femme du confinement familial: "Avant notre emménagement, le territoire de mon épouse se réduisait au logement, au hammam et à des visites familiales au bled (Ghazaouet) où réside sa famille. Étant moi-même pris au travail, c'est mon père qui se chargeait des courses... Dès notre installation, elle s'en est chargée, fréquentant ainsi soit le petit marché de proximité, soit le grand marché hebdomadaire du quartier. »

24 Cette évolution souffre quelques exceptions d'épouses contenues dans les limites domestiques, originaires de zones rurales comme de vieilles cités. Ainsi la mobilité résidentielle est l'occasion du dépassement du territoire domestique au profit du territoire du panier, avec maintien de la fréquentation du hammam et, nouveauté, des douches publiques.

25 À Chteibo, que la mobilité ait relevé du changement de résidence urbaine au sein de l'agglomération ou de l'exode rural, la femme n'a quasiment pas accès à l'espace du panier, encore prérogative du grand-père en cas de cohabitation, du père ou des garçons; elle peut même être exclue de la fréquentation du hammam pour certains néo-urbains. Quel que soit le niveau spatial considéré, le haï ou toute la "ville» périphérique de Chteibo, l'espace public est inexistant: il y a des lieux d'habitat, des lieux de travail, des lieux d'achat... Les cafés eux-mêmes, situés exclusivement sur l'unique grande artère, ne jouent que très faiblement ce rôle.

Qu'il s'agisse des urbains desserrés ou des néo-urbains, le haï est perçu comme l'espace d'interconnaissance et d'entraide, et par là-même réduit au plus à deux rues. Hors de la principale artère des Mawwaline qui concentre l'essentiel des activités commerciales et de services et globalement les catégories sociales les plus favorisées, le haï se structure autour de la mosquée, l'école, l'épicerie, parfois un prestataire de service du type coiffeur ou mécanicien auto ${ }^{15}$ et de plus en plus le hammam.

27 L'entraide semble jouer essentiellement lors de funérailles (Oulima) dont les frais sont pris en charge par les habitants qui se reconnaissent dans le haï, grâce à la pratique de la cotisation qui permet d'acquérir la tente qui devra être dressée devant la maison touchée par le décès et les ustensiles servant à la cuisine des repas servis aux parents et amis appelés à présenter leurs condoléances, le tout étant déposé à la mosquée. La mosquée est elle-même édifiée grâce aux dons du voisinage : un comité informel, à défaut d'association, se charge du suivi de la construction avec l'aide de l'Imam appelé à être rémunéré par l'État. Des urbains en situation de difficulté familiale ou sociale puisent dans cette solidarité un moyen d'intégration et de stabilisation, tel ce garçon de café qui, venant de Savignon, s'installe avec sa famille dans le premier lotissement initié il y a vingt ans et qui, à la suite de vicissitudes familiales (divorce des parents puis conflit), a dû vivre longtemps parmi ses neveux dans un autre lotissement avant de 
fonder un foyer : « Pour moi, la houma est d'abord le lieu de la solidarité, mon mariage a totalement été pris en charge par les voisins de la cinquième tranche. »

La territorialisation est variable selon les catégories sociales et selon les origines. Le sentiment d'appartenance semble très divers. Les néo-urbains sont partagés entre l'ancrage local, porté par les femmes et les jeunes, et l'envie de retour au village avec lequel des liens sont renoués et que l'État encourage à coup de soutiens à la mise en valeur agricole et à l'habitat rural. Pour les habitants venus de la ville d'Oran, Chteibo a constitué d'abord une véritable stabilisation résidentielle après de multiples étapes dues à diverses vicissitudes, et toujours une amélioration de la qualité du logement. Tel habitant, aujourd'hui commerçant prospère sur la rue des Mawwaline, s'est installé ici après être passé par El Hamri, Maraval et Cité Petit ; tel autre employé d'une entreprise publique a dû décohabiter avec ses parents, s'installer chez ses beaux-parents dans un autre quartier avant d'acheter un lot en seconde main et élire domicile à Chteibo en 1996.

Les jeunes de Chteibo, qu'ils soient enfants d'urbains desserrés ou de néo-urbains, sont partagés entre leur appartenance effective à Chteibo et la revendication d'une " oranéité » qui paraît valorisante. Chteibo ayant une image négative, de nombreux jeunes ne se reconnaissent pas dans leur territoire (la maison est celle du père), fréquentent plutôt les cafés oranais, soignent particulièrement leur apparence et préfèrent faire établir leur pièce d'identité dans un des faubourgs oranais ou d'autres communes suburbaines pour ne pas subir les contrôles policiers ou encore le « regard désapprobateur ». Des adultes eux-mêmes peuvent conserver le rattachement administratif antérieur à leur installation ou acquis par leur travail, pour des raisons pratiques : « Tous mes papiers administratifs sont établis à El Karma, je vote à El Karma. J'ai essayé de me faire établir un livret de famille à Sidi Chahmi, cela a pris un an... L'urgence d'une nouvelle municipalité s'impose, d'autant que Chteibo est bien plus grand et plus peuplé que le chef-lieu Sidi Chahmi ", s'indigne un commerçant établi sur la rue des Mawwaline, soutenu par son jeune frère qui vient lui donner un coup de main en dehors de son travail comme gardien de nuit d'une entreprise $:$ «'ai recours au dispensaire d'El Karma pour vacciner mon enfant en raison de la disponibilité du vaccin ». Ce commerçant traduit maladroitement l'idée qu'un centre de pouvoir local est nécessaire pour fonder une appartenance citoyenne. Né dans une commune des monts de l'Ouarsenis, il a grandi à Victor Hugo, banlieue populaire d'Oran, puis à la Jumenterie de Sidi Chahmi avant de s'installer dans un lotissement dédié aux travailleurs qui y étaient employés. Son ancrage est ainsi ancien et définitif mais dédoublé. Cependant, les liens avec son quartier d'enfance oranais sont entièrement rompus : «Tous mes amis ont suivi à Chteibo », reconstituant ainsi des liens de quartier dans un nouveau cadre.

Fils d'un immigrant d'O. 'Aïch arrivé dans les années 1980 à Oran où il a travaillé dans le bâtiment avant de s'installer en 1993 à Haï El Amal, un lotissement dédié aux fonctionnaires, ce coiffeur construit sa propre maison dans un lotissement voisin : pour lui, l'ancrage est total et pourrait être conforté par "plus d'équipements scolaires, sanitaires... ». Ses rapports avec Oran se limitent à ses achats à Mdina Jdida, quartier commercial central.

31 Pour les urbains desserrés, la territorialisation locale est d'autant plus faible que le lieu de travail demeure dans la ville-centre. "Chteibo n'est pas la ville, mais de simples avantages : accès à la propriété et produits à bon marché ", explique cet employé d'une 
entreprise publique. Il conserve des liens privilégiés avec Boulanger, lieu de son travail et de résidence de ses parents comme de ses amis ; son épouse reste liée à Cité Petit où elle séjourne régulièrement chez ses parents, perpétue sa fréquentation de services tels que le hammam. Leurs enfants semblent s'identifier davantage à ces deux quartiers qu'à Chteibo qui n'est considéré que comme une étape, le retour à Oran étant sinon projeté du moins souhaité. De même un couple de cadres moyens de la ville d'Oran arrivant, en 2003, de Saint Eugène où il occupait un logement d'une pièce, a privilégié l'accès à l'habitation individuelle souhaitée à un coût raisonnable "loin des prix délirants d'Oran » et conserve des liens étroits et suivis avec son ancien quartier. À l'inverse, lorsque Chteibo a offert la possibilité de monter une activité commerciale ou de service rentable, les liens avec le quartier d'origine se distendent même quand les enfants y sont nés, au profit d'un sentiment d'appartenance locale.

Chteibo hésite encore entre liens avec la « vraie » ville qu'est Oran et l'attachement à la campagne dont l'image est encore fraîche mais marquée du sceau de la peur. Espace en formation, la qualité du tissu urbain y est médiocre, les équipements structurants sont embryonnaires et très sélectifs, et l'image est négative au point qu'il se dégage, parfois, des discours notamment celui des jeunes une sorte sinon de détestation, du moins de négation du territoire. Le développement de nouvelles filières de promotion immobilière à l'attention des classes moyennes (logement social participatif, locationvente) suscite depuis peu une mobilité qu'il convient de mesurer. Des populations naguère séduites par la maison individuelle (dans n'importe quelles conditions) sont aujourd'hui attirées par les nouvelles résidences d'habitat collectif qui garantissent, malgré une localisation périurbaine, un accès continu aux réseaux techniques et aux services publics et une plus forte intégration à la "ville ». Cette alternative s'offre à tous ces urbains qui, loin de revenir vers leur quartier d'origine, seraient amenés à effectuer une mobilité intrapériurbaine que détermine alors la diversification des segments du marché immobilier.

\section{Conclusion}

Les pratiques et les représentations révèlent dans la société urbaine algérienne un mélange variable d'individualisme et de besoin de sociabilité. Les référents religieux peuvent servir d'alibi à l'expansion du premier face aux normes publiques, mais constituer un support réel à la satisfaction du second.

Qu'il s'agisse des grands ensembles enquêtés, ou des agrégats périurbains tel Chteibo, l'espace public se construit progressivement à l'initiative des habitants eux-mêmes, souvent en réaction à ce qui est considéré comme une agression extérieure, et ne s'ouvrent que marginalement aux femmes. Dans les premiers, le mode de territorialisation semble d'abord passer par un corporatisme jusque-là inhérent au mode d'attribution des logements, que recoupent des solidarités puisées dans les origines régionales, voire dans un passé commun. Il se consolide et s'élargit ensuite, se diversifie à mesure que des équipements se mettent en place et structurent l'espace. Le fait que ces ensembles s'adossent à d'anciens quartiers structurés facilite le processus de sociabilisation. À Chteibo, la genèse du tissu urbain (par tranches de lots) et les modalités de peuplement ont engendré paradoxalement à la fois un puissant individualisme favorisé par l'atmosphère de spéculation persistante et de " débrouille " et la constitution de territoires de voisinage sur une base fréquemment 
communautaire. La sociabilisation est d'autant plus malaisée que le renouvellement par mobilité est un fait quotidien et que les équipements font cruellement défaut: l'interconnaissance n'a que peu de lieux où elle peut s'exprimer, au coin de la rue, ou à proximité de la mosquée.

Dans les deux cas, c'est à un processus d'appropriation territoriale fortement circonscrite dans un périmètre qu'on peut assimiler à une entité de voisinage (quelques blocs, deux ou trois rues), dont l'ouverture sur le quartier est médiocre ou ponctuelle. Les normes sociales qui régissent la visibilité de la femme comme des jeunes sur l'espace public sont par là même très contraignantes, plus dans les agrégats périphériques que dans les grands ensembles. L'explosion de la mobilité pendulaire à laquelle a largement contribué la libéralisation des transports collectifs joue, en ouvrant l'accès à la ville, contre le cloisonnement, par l'anonymat qu'assure la grande ville face aux territoires du quotidien qui demeurent ceux des normes sociales et culturelles.

\section{BIBLIOGRAPHIE}

Bourdieu P., 1980, Le sens pratique, Paris, Éditions de Minuit, 475 p.

Prenant A., Semmoud B., 2006, «Évolution des systèmes migratoires en Algérie ». Communication au colloque sur « La mobilité géographique des populations en Méditerranée occidentale », Grenade, octobre.

Safar Zitoun M., 2006, « Le regard sociologique sur la ville et l'urbain en Algérie : le retournement du paradigme culturaliste colonial ", communication au colloque Chantiers de la recherche en Sciences humaines et sociales sur le Maghreb, IRMC, Tunis.

Semmoud B., 2004, " Perceptions, appropriations et usages des espaces urbains en Algérie », communication au colloque Usages et appropriations de l'espace : entre pratiques et régulations, Annaba (à paraître).

\section{NOTES}

1. Partiellement car la cohabitation de deux ou plusieurs ménages dans un logement n'est pas exceptionnelle, en particulier à Chteibo.

2. Nous avons toutefois averti les interviewés que cette démarche était indépendante de la procédure de régularisation qui relevait des prérogatives des pouvoirs publics.

3. Notre reconnaissance va à $\mathrm{H}$. Hamdaoui et A. Saïd de l'université des sciences et techniques d'Oran ainsi qu'à S. Mokrane, de l'université d'Essenia.

4. Un commerçant, un enseignant du primaire, un gardien de société, un ingénieur, un employé de la poste, un bijoutier et un tôlier.

5. Entraide traditionnelle à l'occasion des travaux agricoles ou de réalisations collectives.

6. Nombreux sont les habitants en déplacement à Oran qui se munissent, selon le cas, de sacs en plastique ou de brosse pour maintenir leurs chaussures propres. 
7. Chteibo est un pôle régional majeur de revente de matériaux de construction divers : ronds à béton, ciment... et le marché quasi unique de produits de récupération notamment de pièces détachées.

8. Cette expression - Celui qui vit à l'ombre de la cohésion collective triomphe du lion - traduit la force des liens de la 'assabiya, ou esprit de corps, tant décrite par Ibn Khaldoun au XIV ${ }^{\mathrm{e}}$ siècle.

9. Tissemsilt est une wilaya voisine de la wilaya de Relizane et ses habitants ont subi autant le terrorisme et ont élu domicile surtout dans les quartiers informels de la commune voisine de Hassi Bou Nif et secondairement à Chteibo.

10. Littéralement: enfants d'oncle. Cette catégorie est en fait celle de Selat Errahim dans la tradition musulmane.

11. L'image négative que renverraient les grands ensembles est exagérée par certains chercheurs qui n'y voient que cloisonnement et enfermement dans le logement : l'Algérien des ZHUN ne se sentirait chez soi ni dans son logement ni dans sa cité.

12. Des habitants de banlieues nouvelles disent appartenir à tel ou tel quartier de la ville-centre.

13. Assemblée populaire communale, équivalent du conseil municipal français.

14. Cette assertion renvoie implicitement à la tradition musulmane qui veut que quiconque met en valeur une terre pour une certaine durée peut se l'approprier.

15. Le mécanicien auto est essentiel du fait de l'importance, à Chteibo, des activités de transport de marchandises.

\section{RÉSUMÉS}

L'article montre, à partir d'enquêtes par entretiens dans des quartiers d'habitat collectif et de lotissements populaires de la périphérie d'Oran, comment les habitants, urbains ou néo-urbains, se territorialisent au quotidien en produisant de la sociabilité fondée sur un mélange subtil et variable d'expérience de la vie urbaine commune et de liens de nature communautaire ou professionnelle. La diversité résulte autant du mode d'accès à l'habitat (relogement, logement de fonction, habitat social, acquisition sur le marché...) que des modalités de peuplement (mobilité résidentielle, exode rural) qui marquent profondément pratiques spatiales et représentations.

The article shows, through interview-based surveys conducted in collective housing neighbourhoods and low-income developments on the outskirts of Oran how the inhabitants, whether urban or neo-urban, become territorialized in their everyday life by producing sociability based on a variety of subtle and variable experience in common urban life and community-related or professional connections. The diversity results both from the way of accessing housing (rehousing, subsidized accommodation, social housing, purchase on the market...) and forms of settlement (residential mobility, rural exodus), which profoundly mark spatial practices and representations.

\section{INDEX}

Index géographique : Algérie

Mots-clés : quotidien, exode rural, habitat, espace urbain, sociabilité, territoire 


\section{AUTEUR}

\section{BOUZIANE SEMMOUD}

Professeur de géographie

Ladyss (Laboratoire dynamiques sociales et recomposition des espaces), CNRS / Univ. de Paris 8 , Département de géographie,

2 rue de la Liberté,

93526 Saint-Denis Cedex 02

bsemmoudz@yahoo.fr 